\title{
AVALIAÇÃO DA INCORPORAÇÃO DE RESÍDUO DE PAPEL EM TELHAS CERÂMICAS *
}

Resumo

\author{
Euzébio Bernabé Zanelato ${ }^{1}$ \\ Jonas Alexandre ${ }^{2}$ \\ Afonso Rangel Garcez Azevedo 3 \\ Markssuel Teixeira Marvila ${ }^{4}$ \\ Sergio Neves Monteiro ${ }^{5}$ \\ Gustavo de Castro Xavier ${ }^{6}$ \\ Lúcio José Terra Petrucci
}

A incorporação de resíduos proveniente de indústrias na construção civil é uma prática cada vez mais comum, pois além de diminuir a quantidade de material descartado no meio ambiente, pode ainda melhorar as propriedades dos materiais empregados na construção civil. A incorporação de resíduos em cerâmica já é realizada com sucesso em incorporações adequadas, inclusive em telhas onde a qualidade exigida pelo produto é superior. Dentre os resíduos com necessidade de destinação encontra-se o resíduo da indústria de papel. O objetivo deste trabalho é avaliar a incorporação do resíduo de papel na fabricação de telhas cerâmicas, foram utilizadas incorporações de 0\%,10\%, 20\% e 30\% para comparação dos resultados. Os materiais utilizados na pesquisa, argila e o resíduo foram caracterizados pelos ensaios de granulometria, análise química e densidade real dos grãos. Para avaliar o melhor teor de incorporação foram confeccionados corpos de prova e realizados os ensaios de resistência à flexão, variação linear e absorção de água. Os resultados indicaram que é viável a utilização da incorporação de $10 \%$ de resíduo, apenas por questões econômicas e ambientais, já que as propriedades pouco se alteraram nesta incorporação.

Palavras-chave: Telhas cerâmicas; Resíduo de papel; Cerâmica vermelha. .

\section{EVALUATION OF THE INCORPORATION OF PAPER RESIDUE IN CERAMIC TILES}

\section{Abstract}

The incorporation of waste from industries in civil construction is an increasingly common practice, as well as reducing the amount of material discarded in the environment, it can improve the properties of the materials used in Civil construction. The incorporation of waste in ceramics is already successfully carried out in suitable incorporations, including in tiles where the quality required by the product is superior. Among the waste that needs to be disposed of is the waste from the paper industry. The objective of this work was to evaluate the incorporation of the paper residue in the ceramic tile fabrication. $0 \%, 10 \%, 20 \%$ and $30 \%$ incorporations were used to compare the results. The materials used in the research, clay and the residue were characterized by the granulometry tests, chemical analysis and real grain density. In order to evaluate the best incorporation content, test specimens were made and the flexural strength, linear variation and water absorption tests were performed. The results indicated that it is feasible to use the incorporation of $10 \%$ of waste, only for economic and environmental reasons, since the properties were little changed in this incorporation.

Keywords: Ceramic tiles; Paper waste; Red ceramic.

Engenheiro Civil, doutorando em Estruturas, LECIV, Universidade Estadual do Norte Fluminense Darcy Ribeiro, Campos dos Goytacazes, RJ, Brasil. 
2 Engenheiro Civil, doutor em Estruturas, LECIV, Universidade Estadual do Norte Fluminense Darcy Ribeiro, Campos dos Goytacazes, RJ, Brasil.

3 Engenheiro Civil, doutorando em Estruturas, LECIV, Universidade Estadual do Norte Fluminense Darcy Ribeiro, Campos dos Goytacazes, RJ, Brasil.

4 Engenheiro Civil, mestrando em Estruturas, LECIV, Universidade Estadual do Norte Fluminense Darcy Ribeiro, Campos dos Goytacazes, RJ, Brasil.

5 Engenheiro Metalúrgico, doutor em Ciência e Engenharia de Materiais, Seção de Engenharia Mecânica e de Materiais, Instituto Militar de Engenharia, Rio de Janeiro, RJ, Brasil.

6 Engenheiro Civil, doutor em Estruturas, LECIV, Universidade Estadual do Norte Fluminense Darcy Ribeiro, Campos dos Goytacazes, RJ, Brasil.

7 Engenheiro Civil, doutor em Engenharia e ciência dos materiais, LAMAV, Universidade Cândido Mendes, Campos dos Goytacazes, RJ, Brasil. 


\section{INTRODUÇÃO}

O município de Campos dos Goytacazes apresenta uma grande reserva de argila, o que motivou o avanço do mercado de cerâmica vermelha na região. No entanto, a grande quantidade de cerâmicas na região não motivou a procura por avanços tecnológicos, e consequentemente, os processos rudimentares usados desde 0 início das atividades continuam sendo utilizados na maioria das cerâmicas.

Outro ponto a ser destacado é a quantidade de cerâmicas que fabricam apenas blocos cerâmicos, assim, a fabricação de telhas é restrita a poucas cerâmicas da região. Isto se deve pelo fato da qualidade inferior das argilas da região [1], que proporcionam peças cerâmicas com qualidade não tão altas, o que prejudica principalmente a fabricação de telhas, já que estas demandam requisitos de qualidade superiores aos dos blocos cerâmicos [2].

Dentre os meios de melhorar as propriedades das peças cerâmicas, a incorporação de resíduo surge como uma medida satisfatória para alcançar as propriedades necessárias para a produção de telhas, sendo economicamente viável além de diminuir o passivo ambiental da indústria que descartaria o resíduo, dentre eles o resíduo de papel.

O objetivo deste trabalho é avaliar a incorporação do resíduo da produção de papel em telhas cerâmicas, assim seria possível dar um destino à um resíduo que seria descartado.

\section{MATERIAIS E MÉTODOS}

A argila utilizada no trabalho foi coletada na cerâmica onde já se fabrica telhas sem a incorporação de resíduo. A argila foi caracterizada pelos ensaios de granulometria, análise química, limites de Atterberg e densidade real dos grãos.

O resíduo da indústria de papel foi coletado diretamente na empresa responsável pelo seu descarte. O resíduo é coletado em forma de lama, assim, o mesmo deve ser secado para a realização dos trabalhos.

Para caracterização do resíduo foram realizados os ensaios de granulometria, análise química e densidade real dos grãos.

Após a caracterização dos materiais procedeu-se a confecção dos corpos de prova cerâmicos com as incorporações de $0 \%, 10 \%, 20 \%$ e $30 \%$. Os corpos de prova foram queimados a temperatura de $750^{\circ} \mathrm{C}$. Foram realizados ensaios de variação linear, resistência à flexão e absorção de água nos corpos de prova confeccionados, conforme recomenda a NBR 15310 [3].

A caracterização do resíduo foi dividida em duas partes: Caracterização física e química. A caracterização física foi constituída pelos ensaios de granulometria, limites de Atterberg e densidade real dos grãos, já a caracterização química foi realizada pelo ensaio de análise química.

A granulometria foi determinada pelo ensaio de acordo com a norma NBR 7181 [4]. Até a peneira ANBT no $200(0,074 \mathrm{~mm})$, os materiais foram classificados pelo peneiramento fino e grosso. Abaixo deste diâmetro feito por sedimentação. No ensaio de sedimentação foi utilizada a substância hexametafosfato de sódio como defloculante. A classificação do solo foi feita de acordo com Casagrande (1942) Unified Soil Classification System. Muito utilizado pelo U.S Bureau of Reclamation (barragens) e U.S. Army Corps of Engineers (aeroportos).

Para realização do ensaio dos limites de Atterberg o solo foi primeiramente destorroado e passado na peneira ABNT no $40(0,42 \mathrm{~mm})$. O Limite de liquidez foi 
realizado conforme NBR 6459 [5], para isso utilizou-se o aparelho de Casagrande. 0 ensaio de limite de plasticidade foi realizado com uma placa esmerilada e foi feito de acordo com a NBR 7180 [6]

A determinação da densidade real dos grãos foi realizada com o picnômetro e executado conforme recomendações das normas NBR 6508 [7] e NBR 6457 [8]

A análise química foi realizada através de Espectroscopia de Energia Dispersiva de Raios X (EDX) no equipamento da Shimadzu EDX-700, onde foram obtidos os elementos constituintes e suas quantidades.

Após a caracterização dos materiais foi realizado os ensaios nas massas cerâmicas produzidas pelos ensaios de Variação linear, Resistência à flexão em 3 pontos e absorção de água. Estes ensaios são importantes para verificação da influência que a adição do resíduo causa nas propriedades de cada um destes ensaios.

Estes ensaios são amplamente utilizados por outros autores para verificação das propriedades das massas cerâmicas [9], [10], [11]. Foram utilizadas doze amostras para cada incorporação em cada um dos ensaios realizados.

\section{RESULTADOS E DISCUSSÃO}

A distribuição granulométrica apresenta similaridades entre os materiais, a soma da fração sílte + argila para o solo utilizado corresponde a $93,6 \%$, enquanto que para o resíduo corresponde a $88,8 \%$. No entanto, o resíduo apresenta maior tamanho de grãos já que sua composição principal é de sílte enquanto que o do solo é de argila.

Tabela 1. Caracterização Química da Massa Cerâmica.

\begin{tabular}{|c|c|c|c|c|c|c|c|c|}
\hline \multirow{3}{*}{ Amostra } & \multicolumn{8}{|c|}{ Porcentagens Granulométricas } \\
\hline & \multicolumn{3}{|c|}{ Pedregulho } & \multicolumn{3}{|c|}{ Areia } & \multirow{2}{*}{ Sílte } & \multirow{2}{*}{ Argila } \\
\hline & Grosso & Médio & Fino & Grossa & Média & Fina & & \\
\hline Solo & - & - & - & 0,3 & 0,2 & 5,9 & 34,7 & 58,9 \\
\hline Resíduo & - & - & - & - & 2,8 & 8,4 & 50,9 & 37,9 \\
\hline
\end{tabular}

A tabela 2 indica os resultados obtidos na análise química da argila e do resíduo. Pode-se verificar grande disparidade entre os materiais em relação aos elementos químicos presentes no mesmo.

Tabela 2. Caracterização Química do Resíduo utilizado.

\begin{tabular}{|c|c|c|c|c|c|c|c|c|c|c|}
\hline & $\mathrm{SiO}_{2}$ & $\mathrm{Fe}_{2} \mathrm{O}_{3}$ & $\mathrm{Al}_{2} \mathrm{O}_{3}$ & $\mathrm{CaO}$ & $\mathrm{MgO}$ & $\mathrm{K}_{2} \mathrm{O}$ & $\mathrm{TiO}_{2}$ & $\mathrm{SO}_{3}$ & $\mathrm{Na}_{2} \mathrm{O}$ & Outros \\
\hline Argila & 59,97 & 9,49 & 12,62 & 4,75 & 1,96 & 5,3 & 0,96 & 0,17 & 2,64 & 2,14 \\
\hline Resíduo & 8,56 & 0,75 & 8,90 & 79,00 & - & 1,16 & 0,35 & 1,11 & - & 0,17 \\
\hline
\end{tabular}

A tabela 3 apresenta os resultados obtidos pelos limites de Atterberg para a argila e de densidade real dos grãos para a argila e o resíduo. 
Tabela 3. Caracterização Química do Resíduo utilizado.

\begin{tabular}{ccc} 
& \multicolumn{2}{c}{ PESQUISA ATUAL } \\
\cline { 2 - 3 } LL & ARGILA & RESÍDUO \\
LP & 71,6 & - \\
IP & 27,7 & - \\
Yg & 23,9 & - \\
USCS & $\mathrm{CH}$ & $\mathrm{SC}$ \\
\hline
\end{tabular}

Após a caracterização dos materiais e confecção dos corpos de prova foram realizados os ensaios nos mesmos. A figura 1 apresenta os resultados obtidos para variação linear. Pode-se observar que o aumento da incorporação de resíduo provoca a diminuição da variação linear. Apenas o teor de 10\% apresentou valores similares ao referência com $0 \%$ de incorporação.

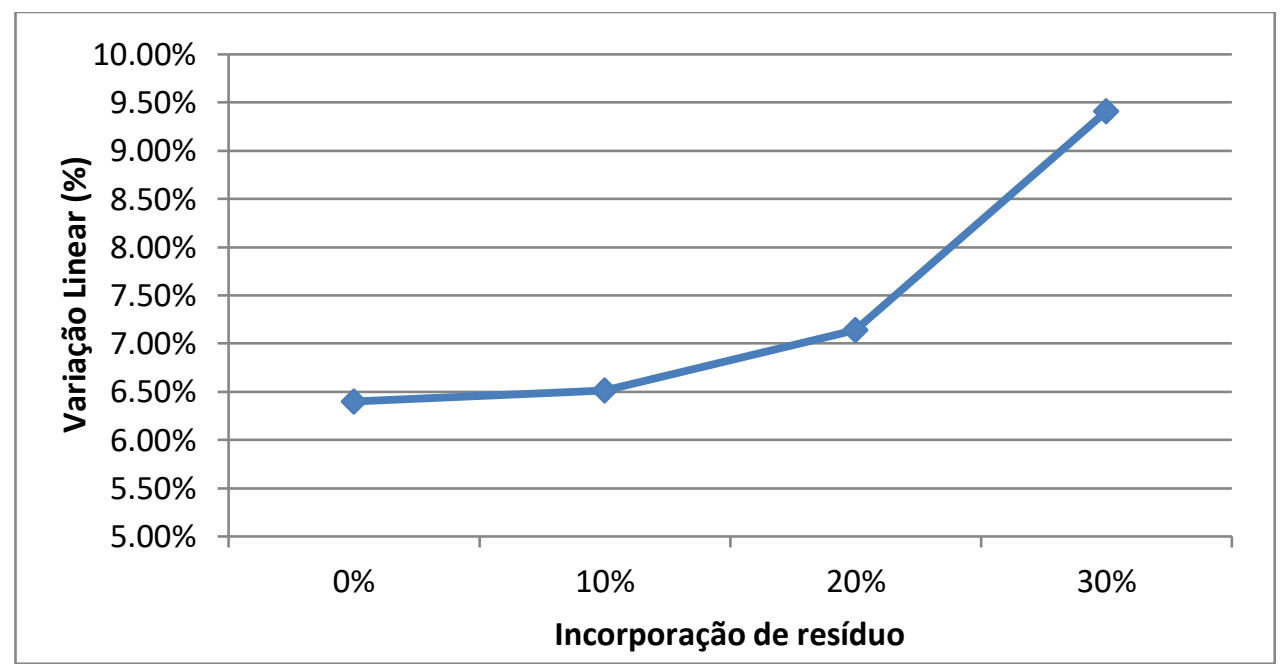

Figura 1. Variação linear.

Outro parâmetro analizado foi a resistência à flexão em 3 pontos, onde os resultados estão indicados na Figura 2. Como pode ser observado, os resultados obtidos pela incorporação de $0 \%$ e $10 \%$ apresentaram novamente resultados similares, no entanto, as incorporações de $20 \%$ e $30 \%$ apresentaram .resistências mecânica inferiores. 


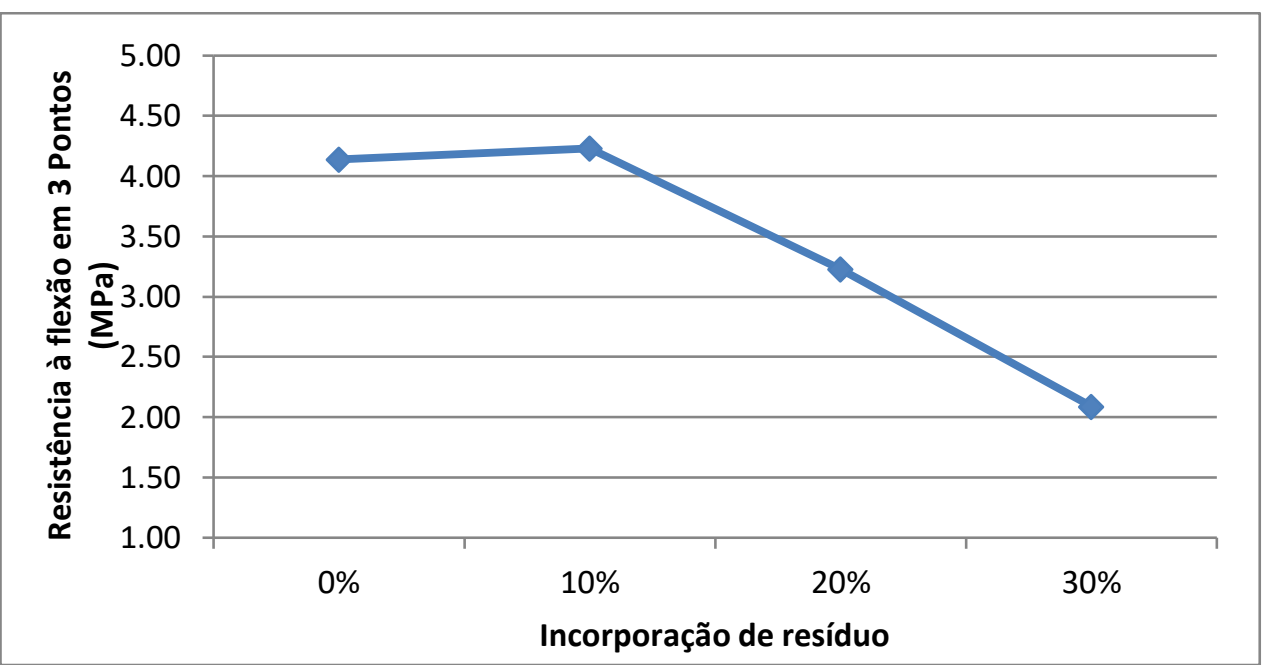

Figura 2. Resistência à flexão em 3 pontos.

Os resultados obtidos no ensaio de absorção de água das peças cerâmicas estão indicados na Figura 3. Pode-se observar que a absorção de água também comprova que a incorporação de $10 \%$ possui propriedades semelhantes à de referência de $0 \%$. Além disso, novamente foi observado um decréscimo na qualidade das peças com o aumento da incorporação a partir dos $10 \%$.

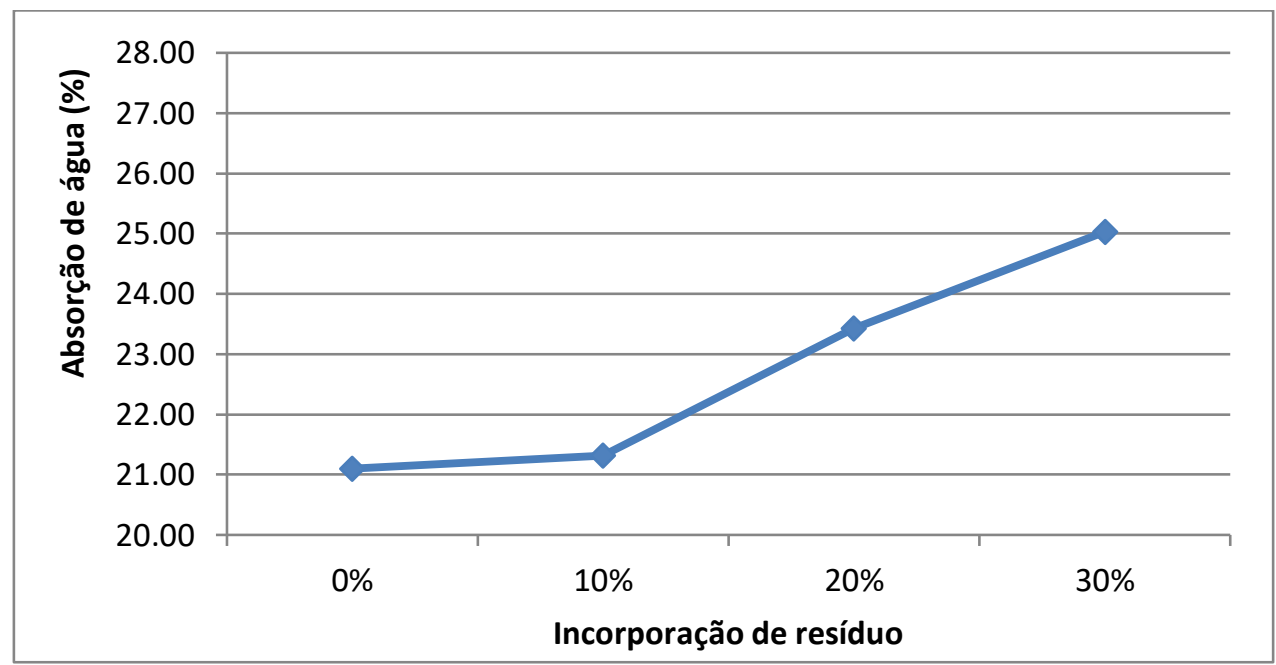

Figura 3. Absorção de água.

\section{CONCLUSÃO}

Pode-se concluir que os resultados obtidos pela incorporação de 10\% são similares ao de referência sem incorporação do resíduo, sendo assim, justifica-se sua incorporação devido aos benefícios econômicos e ambientais resultantes dos mesmos. No entanto, não houve melhora nas propriedades com a adição do resíduo e não é recomendado a incorporação de resíduo em teores acima de 10\% com risco de perda significativas da qualidade das peças.

\section{REFERÊNCIAS}

1 Vieira CMF, Soares TM, Sánchez R, Monteiro SN. Incorporation of granite waste in red ceramics. Materials Science and Engineering A. V 373, 2004. p115-121. 
R. R. Menezes, H. S. Ferreira, G. A. Neves, H. L. Lira, H. C. Ferreira. Use of granite sawing wastes in the production of ceramic bricks and tiles. Journal of the European Ceramic Society. 25, 2005. P1149-1158.

3 ASSOCIAÇÃO BRASILEIRA DE NORMAS TÉCNICAS (2009). ABNT NBR 15310. Componentes cerâmicos - Telhas - Terminologia, requisitos e métodos de ensaio.

4 ASSOCIAÇÃO BRASILEIRA DE NORMAS TÉCNICAS (1988). ABNT NBR 7181. Solo - Análise Granulométrica Conjunta. Rio de Janeiro.

5 ASSOCIAÇÃO BRASILEIRA DE NORMAS TÉCNICAS (1984). ABNT NBR 6459. Solo Determinação do limite de liquidez. Rio de Janeiro

6 ASSOCIAÇÃO BRASILEIRA DE NORMAS TÉCNICAS (1988). ABNT NBR 7180. Solo - Determinação do Limite de Plasticidade. Rio de Janeiro.

7 ASSOCIAÇẤO BRASILEIRA DE NORMAS TÉCNICAS (1984). ABNT NBR 6508. Grãos de solos que passam na peneira de $4,8 \mathrm{~mm}$ - Determinação da massa específica. Rio de Janeiro.

8 ASSOCIAÇÃO BRASILEIRA DE NORMAS TÉCNICAS (1986). ABNT NBR 6457. Amostras de solo - Preparação para ensaios de compactação e ensaios de caracterização. Rio de Janeiro.

9 Souza AJ; PINHEIRO BCA; HOLANDA JNF. Efeito da adição de resíduo de rocha ornamental nas propriedades tecnológicas e microestrutura de piso cerâmico vitrificado. Cerâmica, São Paulo, v. 57, n. 342, p. 212-218, June 2011

10 Rodrigues DV, Xavier GC, Saboya F, Maia PCA, Alexandre, J. Durabilidade de peças cerâmicas vermelhas com adição de rejeito de rocha ornamental isenta de granalha. Cerâmica, São Paulo, v. 58, n. 347, p. 286-293, Sept. 2012

11 Areias IOR, Vieira CMF, Manhães RST, Intorne AC. (2017). Incorporação de lodo da estação de tratamento de esgoto (ETE) em cerâmica vermelha. Cerâmica, 63(367), 343-349 